\title{
Comparison of Zinc,Vitamin A \&D Serum Levels Between Children With Respiratory Tract Infection And Healthy Individuals in two Iranian Referral Hospitals
}

\author{
Mohammad Reza Shokrollahi ${ }^{1}$, Zahra Movahedi ${ }^{1}$, Shima Javadi Nia ${ }^{2}$, \\ Hosein Masoumi Asl ${ }^{3}$, Hosein Heydari ${ }^{1}$, Azardokht Tabatabaei ${ }^{2, *}$ \\ ${ }^{I}$ Department Of Pediatric Infectious Diseases, School Of Medicine, Qom University Of Medical Sciences, Qom, \\ Iran \\ ${ }^{2}$ Research Center Of Pediatric Infectious Diseases, Hazrat-E-Rasool Akram Hospital, Iran University Of \\ Medical Sciences, Tehran, Iran. \\ ${ }^{3}$ Center for Communicable Diseases Control, Ministry Of Health And Medical Education, Tehran, Iran
}

\begin{abstract}
Background and objective:Different factors influence the severity of respiratory infection such as causative pathogen and the host immune response. Vitamins and micronutrients play important roles in supporting immune system, and their homeostasis have a crucial importance in response to infections. Nutritional deprivation both for micronutrients and vitamins especially Zinc and vitamins A and D are particularly common in children of Middle East and Asia. This problem arises from their poor and inadequate diet besides the loss of nutrients in course of recurrent infections. Materials and methods:This was a cross-sectional trial on 25 patients, admitted to pediatric wards of two referral children hospitals in Tehran, Iran, due to acute respiratory tract infection, who were compared to 40 other patients admitted to surgical ward for elective surgery. $72 \%$ of the patients were male and $28 \%$ female. The average age was 2.17years. Serum levels of Zinc, vitamins A and D were measured in both groups using atomic methods, ELISA and spectrophotometry, respectively.

Results:Despite the lower levels of vitamins $A$ and $D$ in cases than controls, the difference was not significant ( $p=0.4$ and $p=0.1$, respectively).

However, serum levels of Zinc were significantly lower in cases than controls ( $p=0.002)$.

Conclusion:According to our trial, it seems administration of supplementary zinc and vitamin A might reduce patients' vulnerability to respiratory tract infection. There was no such indication in regards for vitamin $D$ supplementation, which could be due to the relatively low levels of this vitamin in the whole population.
\end{abstract}

Key words: vitamin A, vitamin D, Zinc, respiratory tract infection

\section{Introduction}

The mortality rate of respiratory tract infection is about 2 million children annuálly. Most of these children live in developing countries like Iran(1). Even in developed countries, the prevalence of these infections is very high. Around 19 to $27 \%$ of annual hospitalizations for children younger than 15 is caused by acute respiratory tract infection $(2,3)$. There is not a precise statistics about the situation in our country.

The severity of respiratory tract infection is determined by different factors such as the causative pathogen and the host immune response. Vitamins and micronutrients plays important roles in supporting human immune system, and their homeostasis has a crucial importance in response to infections(4). Nutritional deprivation both for micronutrients and vitamins especially Zinc, vitamins A and D are particularly common in children of Middle East and Asia. This problem arises from their poor and inadequate diet besides the loss of nutrients in course of recurrent infections (5).

Many interventional trials had investigated vitamin A positive influence on respiratory tract infections. According to their results high doses of vitamin A reduces the number and severity of acute non measles respiratory infections $(6,7)$.

There is no solid proof in favor of the part played by Zinc in infections. Two published clinical trials revealed no specific relation between zinc and infection (8). On the other hand, a comparatively large clinical trial demonstrated a $45 \%$ decrease in pneumonia prevalence with Zinc supplementation (9).

Besides rickets, other complications have recently been reported as results of Vitamin D deficiency. The relationship between vitamin D and acute respiratory tract infections was shown in a cross-matched prospective trial (10) and other case-control studies $(11,12)$. Clinical vitamin D deficiency and rickets increases the risk of 
pneumonia in Ethiopian children younger than 5 years by 13 times. Another study in Yemen showed that $50 \%$ of children hospitalized due to acute respiratory infections had also vitamin D deficiency.

Despite the obvious fact that nutritional deprivation aggravates the clinical course of illness and prolongs recovery (13), there is still a shortage of testament in favor of such correlation with respiratory tract infection severity.

Respiratory tract infection is one of the main reasons for hospitalization in children. The role played by micronutrients in infections was shown in Iranian children before.

Purpose of this trial is to determine the correlation between serum levels of zinc, vitamin A and D in children suffering from acute respiratory infection in contrast to healthy individuals.

\section{Materials and methods}

This trial was carried out on 25 children hospitalized due to lower respiratory tract infection (pneumonia) in two referral children hospitals and another 40 healthy individuals in the period of 2013 to 2015. Patients were between 6 months and 5 years old, $72 \%$ of them were male and $28 \%$ were female.

The trial was performed after being credited by Ethics Committee of Pediatric Infectious Diseases Research Center of Iran University of Medical Sciences as to be compatible with Helsinki Regulations. An informed consent was obtained from every patient.

First, pneumonia diagnosis was established and documented based on WHO criteria (3), then a questionnaire on personal information and patients` medical history and imaging results was filled out.

Cases: children diagnosed with lower respiratory tract infection based on clinical presentation, physical examination and imaging.

Diagnostic criteria for lower respiratory tract infection included cough, fever, abnormal breath sounds (rale, rhonchi, crackle), increased respiratory rate according to age (more than 50 in 5 to 12 month-olds, more than 40 in 4 to 13 year-olds).

5 CCs of blood clot were collected from both groups of patients and the serum was separated and kept in freezer. Zinc, vitamins A and D levels were measured for all patients. The results were reported according to the standard levels indicated on laboratory kits.

To measure Zinc levels we used atomic absorption spectrophotometer technique using GBC aventa apparatus manufactured in Australia. According to kit's standard Zinc levels equal or higher than 65 microgramsin deciliter were considered normal.

Vitamin A (Retinol) levels were measured by HPLC craft. Normal levels of vitamin A according to kit's standard (categorized by age) were equal or higher than 20 micrograms in deciliter.

Vitamin D (25 OH Vit D) was measured by Radioimmunoassay (Diasorin, stillwater, MN USA; kit). Vitamin D levels equal or higher than 7.5 nanograms in milliliter were considered normal.

Collected data and lab results were analyzed using T-test, Chi square and other tests by SPSS software. $P$ values lower than 0.05 were considered significant.

\section{Results}

Vitamin A levels in cases were lower than controls; however, the difference was not significant (P value $=0.4)$. The same results were reported for vitamin $\mathrm{D}(\mathrm{P}$ value $=0.1)$. Zinc levels, however, were significantly lower in patients than in controls $(\mathrm{P}$ value $=0.002)$.

Table 1. Comparison of micronutrient levels in patients and healthy individuals

\begin{tabular}{|c|c|c|c|c|c|c|}
\hline \multirow{2}{*}{$\begin{array}{l}\text { micronutrients } \\
\text { Respiratory tract } \\
\text { infection }\end{array}$} & \multicolumn{2}{|c|}{ Vit A mcg/dl } & \multicolumn{2}{|c|}{ Vit D ng/ ml } & \multicolumn{2}{|c|}{ Zinc mcg/dl } \\
\hline & patient & Control & Patient & control & patient & control \\
\hline Average & 0.27 & 0.41 & 35.37 & 45.7 & 87.93 & 106.9 \\
\hline Standard deviation & 0.169 & 0.202 & 34.68 & 21.05 & 23.34 & 23.7 \\
\hline $\mathrm{P}$ value & \multicolumn{2}{|l|}{0.4} & \multicolumn{2}{|l|}{0.1} & \multicolumn{2}{|c|}{0.002} \\
\hline
\end{tabular}

\section{Discussion and conclusion:}

Vitamin D is produced through photolytic reaction caused by ultraviolet rays in skin from 7dehydrocholesterol. This vitamin is also naturally digested with our food. The end metabolite produced in skin and the vitamin absorbed from intestines should be hydroxylated in liver to 25-hydroxyvitamin D. This product hydroxylated in kidneys to 1,25 hydroxyvitamin $\mathrm{D}$ which is the active form of the vitamin. Main role of vitamin $\mathrm{D}$ is to regulate the homeostasis of calcium and phosphorus, which are essential in bone mineralization. To assess vitamin D in body 25-hydroxyvitamin D in serum is measured. Vitamin D deficiency is associated with increased parathormon secretion, when 25hydroxyvitamin $\mathrm{D}$ level is lower than 78-100 $\mathrm{nm}$ in liter parathormone secretion increases. 25hydroxyvitamin D levels lower than $80 \mathrm{~nm}$ in liter is generally believed to be deficient. Another new and different action which recently ascribed forvitamin D is its anti-proliferative and 
apoptotic effect on prostate cancerous cells. Vitamin D has also the main role in connection between activating toll-like receptors and antimicrobial response of innate immunity(10).

It is demonstrated that vitamin D effects on monocytes, macrophages, Arnold had even gone further and concluded that appropriate vitamin D levels have protective effects on TB skin test changes from negative to positive(14).

Hewison showed that the effect of vit $\mathrm{D}$ on immune system is not done viaclassic endocrine pathway but comparatively paracrine and intracrine pathway are also responsible for some of its regulatory effects (15).

Mueheilsin proved that vitamin $\mathrm{D}_{3}$ along with parathyroid hormone increases cathelicidin and immune defenses synergically and its deficiency in mice increases the risk of infections(16).

Sterling revealed deficiency of active vitamin D in renal failure to be the reason for immune (17).

Korthals in a study carried out in the Netherlands concluded that the seasonal prevalence of TB to be a result of decreased serum vitamin D levels (18), reduced serum level of vit D also was found in refugee children with latent and TB infection(19).

Low levels of vitamin D in respiratory tract infections will have taken longer to recover (20) and furthermore Laaksi and barri concluded that there is a relationship between vitamin D deficiency and lower respiratory tract infection and therefore vitamin $\mathrm{D}$ supplementation has apreventive effect on respiratory tract infection $(21,22,23)$.

Other trials showed that children with severe lower respiratory tract infection who need assistant ventilation had lower levels of serum vitamin D $(24,25)$.

Oduwole showed that not only vitamin D deficiency but also its insufficiency can leads to higher prevalence of infection especially pneumonia in children (26). Talk of the protective effects of vitamin D went so far as to Morales suggesting that its administration during pregnancy would decrease the risk of lower respiratory tract infection in the newborn`s first year of life (27)and leis demonstrated in his trial that vitamin D levels higher than recommended dose in children younger than 5 years could be protective against lower respiratory tract infections (28).

On the other hand, a trial in Romania reported that despite very low levels of vitamin D in women no correlation with respiratory infections is perceived (29).

An animal trial performed by Sacco was not able to reveal the relation between RSV infection and vitamin Dserum level (30).

Both groups in our trial had vitamin D levels lower than normal with the average of $35 \mathrm{ng}$ in cases and $45 \mathrm{ng}$ in controls. Although the amount is much lower in the cases it still is not of significant importance. Therefore our trial was much like Romania trial, which could have happened as a result of nationwide low levels of vitamin D.

Vitamin A deficiency is one the major nutritional deficiencies seen in preschool children in developing countries. Various studies had proved that vitamin supplementation to these children`s diet would reduce their mortality rate by almost $23 \%$, even though the mechanism is still unknown (31). It is believed that such benefits are seen through its ability to reduce the prevalence of infectious diseases. This hypothesis was proposed based on 3 different studies. The first one was carried out on animals, which proved that vitamin A deficiency would have diminished resistance against infections. The second trial showed that vitamin A deficiency have made patients more susceptible to respiratory and gastrointestinal infections. The third trial in Indonesia showed that prescribing high doses of vitamin A to preschool children decreased mortality by $34 \%$ (31).

Vitamin A deficiency compromises both cellular and humoral immunity in mucus. Dendritic cell regulation is the main mechanism through which vitamin A deficiency affects mucosal immune response to infection (32).

Mathew et al reported pneumonia as the cause of $1 / 4$ of deaths in children younger than 5 in India. They also reported even higher mortality rates in younger children and those suffering from malnutrition. In their survey, there is little evidence in support of preventive or curative effects of vitamin A in pneumonia (33).

Abba et al showed that there is no evidence indicating the role of supplementary vitamin A to alter mortality rate or sputum culture results in patients under treatment for TB (34).

In another trial on children younger than 6 months Chen noticed that the prevalence of respiratory diseases, cough, runny nose, and fever are lower in children receiving vitamin A, iron, thiamin, riboflavin, folic acid, niacin amid, zinc and calcium than those receiving only vitamin $\mathrm{A}$ or vitamin $\mathrm{A}$ and iron(35).

He also performed another study in children younger than 7 , concluding that vitamin A in children with malnutrition and low weight reduced lower respiratory tract infection; while in normal individuals it increased the rate. Therefore, supplementary vitamin A is recommended only in those children with malnutrition and low weight(36).

On the other hand some trials show the efficacy of vitamin A in respiratory infections, e.g. vitamin A reduces the mortality of measles and its administration in children older than 6 months reduces its prevalence. Diness et alinvestigated the effects of vitamin A administration after birth in Guinea-Bissau. They followed 
4183 28-day old children until they turned 6 months old. In 165 cases with measles they noticed shorter hospitalization in those receiving vitamin A and mortality in boys (and not girls) compared to the control group (37). In another Meta-analysis done by Sudfeld on 25 studies, he deduced that vitamin A in children reduces prevalence of measles(38).

Irlam performed another Meta-analysis on 30 trials and discovered that adding vitamin A to adults`diet did not have any influence on HIV progression but in African children it reduced HIV mortality along with mortality related to diarrhea and pulmonary infection (39).

There are many other studies indicating the same findings. Lou et al proved in a trial that vitamin A deficiency besides increasing respiratory infection exacerbated wheeze in infants, so that its duration and severity were associated with vitamin A deficiency (40). Cameron and colleges investigated vitamin A levels in 305 children younger than 5 years and found out that vitamin A deficiency was an important risk factor in acute otitis media and lower respiratory infection (41).

Qian examined the relation between vitamin $A$ and immunoglobulin $G$ in recurrent respiratory infections in children and noticed that the serum levels of immunoglobulin $G$ subclasses in children with vitamin A deficiency was lower (42).

In our trial as well, vitamin A levels in children with respiratory tract infection was lower than the control group. This indicates the role of vitamin A deficiency in respiratory infections and that its correction might prevent the children from getting infected or reduce its severity.

Zinc has many different parts in body metabolism. Zinc deficiency physiologic signs and symptoms are associated with various biochemical functions. As soon as dietary zinc amounts reduce, internal zinc consumption reduces to compensate for the decrease.If the balance does not recover, zinc would be moved from internal reserves. These reserves are unknown but it seems like all cells have small amounts of zinc in Golgi apparatus or other organelles as reserve. Plasma levels of zinc will diminish drastically with severe zinc deficiency and slowly with minor deficiencies. Moreover, zinc levels will also reduce in some situations like infection, trauma, stress, steroid use, and after meals(43).

Mathew et al found out pneumonia as the cause for $1 / 4$ of children under 5 year mortalityduring a trial in India. They also reported higher risk of mortality in younger children and those with malnutrition. In their trial there was not enough proof in favor of preventive or therapeutic effects of zinc in pneumonia (33).

In another trial performed by Lawson on 350 Nigerian patients with positive smear of TB who were older than 15 years old showed that zinc or zinc-vitamin A supplementation has no effect on patient mortality (44). Furthermore, Abba did not find any proof indicating that zinc supplementation to TB patients` diet could decrease mortality rate and alter sputum smear results in his review of 12 trials (35).

Das reviewed 7 clinical trials on children younger than 5 years with acute lower respiratory infection in developing countries and found no difference between the recovery time and hospitalization period in those receiving zinc and the controls (45). On the other hand, cartasuria et al investigated the effects of adding zinc to diet before and after adding vitamin A to diet of children between 2-5 years old in Indonesia. He concluded that supplementary zinc and vitamin A in preschool children diminished the number of days with respiratory infection (46). Bhutta and colleagues found out that adding zinc to children younger than 5 years' diet reduces the prevalence of pneumonia (8).

In a similar study, Dr Ehsani pour et al investigated serum levels of zinc in 142 children between 3 to 60 months old without malnutrition suffering from pneumonia with 142 healthy individuals. Serum levels of zinc in those with pneumonia were significantly lower than controls (47).

Similarly in our trial, the difference of zinc levels between the two groups were considerable, which implied the reduction could happen during respiratory infection or the low zinc levels could make the child vulnerable against the infection.

\section{Conclusion}

According to this trial, it seems that adding vitamin A and zinc to children's diet would reduce the prevalence of respiratory infection. No difference was noticed with different levels of vitamin $\mathrm{D}$, which could be the result of its nationwide deficiency.

\section{Refrencess}

[1]. Behrman RE,Kliegman RM. Fever Without a Focus. Nelson Text book of Pediatrics. Saunders, Philadelphia.2016:841-846.

[2]. Schweich PJ. Fever in young infants. In MCMillan JA principles \&practice.Lippincot Williams \&Wilkins.2006:701702.

[3]. Bennett RT,Mazzaccaro RJ, Chopra N, Melman A, Franco I. Suppression of ... A and E in ascending pyelonephritis in rats. J Urol. 1999; 161:1681-4.

[4]. Black MM. Micronutrient deficiencies and cognitive Functioning. J Nutr 2003 ; 133(supple 2):3927-31S

[5]. ShankarAH, prasadAS. Zinc and immune function : the biological basis of altered resistance to infection . Am J Clin Nute $1998 ; 68(447$ S-63S) 
[6]. Stansfield SK, Pierre-Louise M, Lerebours G, Augustin A. Vitamin A supplementation and increased prevalence of childhood and acute respiratory infections. Lancet 1993;342:578-81 .

[7]. Dibley MJ, Sadjmin T, Kjolhede CL , Moulton LH . Vitamin A supplementation fails to reduce incidence of respiratory Infection illness and diarrhea in pre school-age Indonesian children. J Nutr 1996;126

[8]. Bhutta ZA, Black RE Brown KN , Gardner JM , Gore S , Hidayat A, et al . Zinc investigators' Collaborative Group . Prevention of diarrhea and pneumonia by supplementation in children in developing countries : pooled analysis of randomized controlled trials . J Pediatr 1999;155:689-97

[9]. Sazwal S, Black RE, Jalla S, Mazumdar S, Sinha A, Bhan MK . Zinc supplementation reduces the incidence of acute lower respiratory Infection in infans and preschool children : a double -blind, controlled trial . Pediatrics 1998;102:1-5

[10]. Laaksi I, Ruohola JP , Tuohimaa P, et al . An association of serum vitamin D concentration $40 \mathrm{~mol} / \mathrm{L}$ with respiratory Infection in young finish men .

[11]. Wayse V, Yousafzai A, Mogale K, Filteau S . Association of subclinical vitamin D deficiency with server acute respiratory Infection in Indian children under 5y . Eur J Clin Nutr . 2004; 58(4);563-567.

[12]. Karatekin G, Kaya A, Salihoglu O , Balci H, Nuhoglu A. Association of subclinical vitamin D deficiency in newborns with acute lower respiratory Infection and their mothers [published online November 21,2007] . Eur J Clin Nutr.

[13]. Cuevas LE, Koyanagi A . Zinc and infection : a review . Ann Trop Paediatr 2005; 149-60

[14]. Arnedo-Pena A, Juan-Cerdán JV, Romeu-Garcia A, Garcia-Ferrer D, Holguín-Gómez R, et al Latent tuberculosis infection, tuberculin skin test and vitamin D status in contacts of tuberculosis patients: a cross-sectional and case-control study.. BMC Infect Dis, 2011 Dec 15;11:349

[15]. Hewison M. Vit D and immune function: autocrine, paracrine or endocrine? Scand J Clin Lab Invest Suppl. 2012;243:92-102.

[16]. Muehleisen B, Bikle DD, Aguilera C, Burton DW, Sen GL, et al. PTH/PTHrP and Vitamin D Control Antimicrobial Peptide Expression and Susceptibility to Bacterial Skin Infection. Science Transl Med. 2012 May 23;4(135):135ra66

[17]. Sterling KA, Eftekhari P, Girndt M, Kimmel PL, Raj DS. The immunoregulatory function of vitamin D: implications in chronic kidney disease.Nat Rev Nephrol. 2012 May 22;8(7):403-12

[18]. Korthals Altes H, Kremer K, Erkens C, van Soolingen D, Wallinga J.Tuberculosis seasonality in the Netherlands differs between natives and non-natives: a role for vitamin D deficiency? Int J Tuberc Lung Dis. 2012 may; 16(5): 639-44

[19]. Gray K, Wood N, Gunasekera H, Sheikh M, Hazelton B, Barzi F, Isaacs D. Vit D and tuberfculosis status in refugee children. Pediatr Infect Dis J. 2012; may; 31(5): 521-3

[20]. Laaksi I, Ruohola JP, Tuohimaa P, Auvinen A, Haataja R, et al.An association of serum vitamin D concentration $<40 \mathrm{nmol} / 1 \mathrm{with}$ acute respiratory tract infection. Am J Clin Nutr 2007; 86:714-7

[21]. Laaksi I. Vitamin D and respiratory infection in adults. Proc Nutr soc. 2012 Feb; 71(1):90-7

[22]. Berry DJ, Hesketh K, Power C, Hyppönen E.Vitamin D status has a linear association with seasonal infections and lung function in British adults. Br J Nutr. 2011 Nov; 106(9): 1433-40

[23]. Tsujimoto Y, Tahara H, Shoji T, Emoto M, Koyama H, et al. Active vit D and acute respiratory infection in dialysis patients. Clin J Am Soc Nephrol. 2011 Jun; 6(6): 136-7

[24]. Inamo Y, Hasegawa M, Saito K, Hayashi R, Ishikawa T, et al. Serum vitamin D concentrations and associated severity of acute lower respiratory tract infections in Japanese hospitalized children. Pediatr Int 2011 Apr; 53(2): 199-201

[25]. McNally JD, Leis K, Matheson LA, Karuananyake C, Sankaran K, Rosenberg AM.. Vitamin D deficiency in young children with severe acute lower respiratory infection.Pediatr Pulmonol. 2009 Oct;44(10):981-8.

[26]. Oduwole AO, Renner JK, Disu E, Ibitoye E, Emokpae E.Relationship between vitamin D levels and outcome of pneumonia in children.West Afr J Med. 2010 Nov-Dec;29(6):373-8.

[27]. Morales E, Romieu I, Guerra S, Ballester F, Rebagliato M et al. Maternal vitamin D status in pregnancy and risk of lower respiratory tract infections, wheezing, and asthma in offspring. Epidemiology 2012 Jan;23(1):64-71

[28]. Leis KS, McNally JD, Montgomery MR, Sankaran K, Karunanayake C, Rosenberg AM.. Vitamin D intake in young children with acute lower respiratory infection. Zhonogguo Dang Dai Er Ke Za Zhi 2012 Jan; 14(1):1-6

[29]. Porojnicu AC, Moroti-Constantinescu R, Laslau A, Lagunova Z, Dahlback A, et al. Vitamin D status in healthy Romanian caregivers and risk of respiratory infections.Public Health Nutr. 2012 Nov;15(11):2157-62.

[30]. Sacco RE, Nonnecke BJ, Palmer MV, Waters WR, Lippolis JD, Reinhardt TA.. Differential expression of cytokines in response to respiratory syncytial virus infection of calves with high or low circulating 25-hydroxyvitamin D3. PloS One.2012;7(3)

[31]. Dibley MJ, Sadjimin T, Kjolhede CL, Moulton LH. Vitamin A supplementation fails to reduce incidence of acute respiratory illness and diarrhea in preschool-age Indonesian children.J Nutr. 1996 Feb;126(2):434-42.

[32]. Yang Y. Effects of vitamin A deficiency on mucosal immunity. Nutrition 2011Feb;27(2):227-32

[33]. Mathew JL, Patwari AK, Gupta P, Shah D, Gera T, Gogia S, et al. Acute respiratory infection and pneumonia in India: a systematic review of literature for advocacy and action: UNICEF-PHFI series on newborn and child health, India. Indian Pediatr 2011;48(3):191-218.

[34]. Abba K, Sudarsanam TD, Grobler L, Volmink J. Nutritional supplements for people being treated for active tuberculosis. Cochrane Database Syst Rev 2008;(4):CD006086.

[35]. Chen K1, Zhang X, Li TY, Chen L, Wei XP, Qu P, Liu YX. Effect of vitamin A, vitamin A plus iron and multiple micronutrientfortified seasoning powder on infectious morbidity of preschool children.Nutrition. 2011 Apr;27(4):428-34.

[36]. Chen H, Zhuo Q, Yuan W, Wang J, Wu T. Vitamin A for preventing acute lower respiratory tract infections in children up to seven years of age. Cochrane Database Syst Rev 2008;(1):CD006090.

[37]. Diness BR, Christoffersen D, Pedersen UB, Rodrigues A, Fischer TK, Andersen A et al. The Effect of High-Dose Vitamin A Supplementation Given with Bacille Calmette-Guérin Vaccine at Birth on Infant Rotavirus Infection and Diarrhea: A Randomized Prospective Study from Guinea-Bissau.The Journal of Infectious Diseases, Volume 202, Issue Supplement 1,S243S251.

[38]. Sudfeld CR1, Navar AM, Halsey NA. Effectiveness of measles vaccination and vitamin A treatment. Int J Epidemiol. 2010 Apr;39 Suppl 1:i48-55

[39]. Irlam JH, Visser MM, Rollins NN, Siegfried N. Micronutrient supplementation in children and adults with HIV infection.Cochrane Database Syst Rev. 2010 Dec 8;(12):CD003650.

[40]. Luo ZX, Liu EM, Luo J, Li FR, Li SB, Zeng FQ, Qu P, Fu Z, Li TY. Vitamin A deficiency and wheezing.World J Pediatr. 2010 Feb;6(1):81-4

[41]. Cameron C, Dallaire F, Vézina C, Muckle G, Bruneau S, Ayotte P, et al. Neonatal vitamin A deficiency and its impact on acute respiratory infections among preschool Inuit children. Can J Public Health 2008;99(2):102-6. 
[42]. Qian L, Lu JR. Serum levels of IgG subclasses and vitamin A in children with recurrent respiratory tract infection. Zhongguo Dang Dai Er Ke Za Zhi 2007;9(6):557-8.

[43]. King JC. Zinc: an essential but elusive nutrient. Am J Clin Nutr 2011;94(2):679S-84S.

[44]. Lawson L, Thacher TD, Yassin MA, Onuoha NA, Usman A, Emenyonu NE, et al. Randomized controlled trial of zinc and vitamin A as co-adjuvants for the treatment of pulmonary tuberculosis. Trop Med Int Health 2010;15(12):1481-90.

[45]. Das RR, Singh M, Shafiq N. Short-term therapeutic role of zinc in children < 5 years of age hospitalised for severe acute lower respiratory tract infection. Paediatr Respir Rev 2012;13(3):184-91.

[46]. Kartasurya MI, Ahmed F, Subagio HW, Rahfiludin MZ, Marks GC. Zinc combined with vitamin A reduces upper respiratory tract infection morbidity in a randomised trial in preschool children in Indonesia. Br J Nutr 2012;108(12):2251-60

[47]. Ehsanipour F, Harandi V, Jalali K. The Survey of Serum Zinc Level in Children with Pneumonia,RJMS 2009, 16(62): 21-25 